\title{
Ultrasonographic findings in dogs with hyperammonemia: 90 cases (2000-2002)
}

\author{
Viktor Szatmári, DVM; Jan Rothuizen, DVM, PhD; Ted S. G. A. M. van den Ingh, DVM, PhD; \\ Frederik J. van Sluijs, DVM, PhD; George Voorhout, DVM, PhD
}

\section{Objective-To determine ultrasonographic abnormal- ities in dogs with hyperammonemia.}

Design-Retrospective study.

Animals - 90 client-owned dogs with hyperammonemia.

Procedure-Ultrasonography of the abdominal vessels and organs was performed in a systematic way. Dogs in which the ultrasonographic diagnosis was a congenital portosystemic shunt were included only if they underwent laparotomy or necropsy. Dogs in which the abdominal vasculature appeared normal and dogs in which the ultrasonographic diagnosis was acquired portosystemic shunts and portal hypertension were included only if liver biopsy specimens were submitted for histologic examination.

Results-Ultrasonography excluded portosystemic shunting in 11 dogs. Acquired portosystemic shunts were found in 17 dogs, of which 3 had arterioportal fistulae and 14 had other hepatic abnormalities. Congenital portosystemic shunts were found in 61 dogs, of which 19 had intrahepatic shunts and 42 had extrahepatic shunts. Intrahepatic shunts originated from the left portal branch in 14 dogs and the right portal branch in 5. Extrahepatic shunts originated from the splenic vein, the right gastric vein, or both and entered the caudal vena cava or the thorax. Ultrasonography revealed splenic-caval shunts in 24 dogs, right gastric-caval shunts in 9 dogs, splenic-azygos shunts in 8 dogs, and a right gastric-azygos shunt in 1 dog.

Conclusions and Clinical Relevance-Results suggest that ultrasonography is a reliable diagnostic method to noninvasively characterize the underlying disease in dogs with hyperammonemia. A dilated left testicular or ovarian vein was a reliable indicator of acquired portosystemic shunts. (J Am Vet Med Assoc 2004;224:717-727)

$\mathrm{H}$ igh blood ammonia concentrations in dogs are usually the result of 1 of 3 well-defined conditions: acquired portosystemic shunts (APSSs), congenital portosystemic shunts (CPSSs), and urea cycle enzyme deficiency, a rarely detected congenital disease characterized by decreased activity of 1 or more of the

From the Division of Diagnostic Imaging (Szatmári, Voorhout) and the Departments of Clinical Sciences of Companion Animals (Rothuizen, van Sluijs) and Pathology (van den Ingh), Faculty of Veterinary Medicine, Utrecht University, Yalelaan 10, 3584 CM, Utrecht, The Netherlands.

Dr. Szatmári was supported by the Hungarian State Eötvös Scholarship

Presented in part at the WSAVA-FECAVA-AVEPA Veterinary Congress in Granada, Spain, October 2002.

Address correspondence to Dr. Szatmári. enzymes that transform ammonia into urea. ${ }^{1,2}$ Portosystemic shunting occurs when anomalous veins allow portal blood to enter the systemic veins directly, without first flowing through the hepatic sinusoids. Acquired portosystemic shunts develop as a result of sustained hepatic or prehepatic portal hypertension and represent enlargement of rudimentary extrahepatic vessels through which no blood normally passes. ${ }^{2,3}$ Shunt formation is a compensatory mechanism to maintain normal portal pressure by allowing portal blood to drain into the lower pressure systemic veins. Because causes that lead to APSS formation can be congenital (eg, arterioportal fistulae) or acquired (eg, hepatic cirrhosis), these shunts can develop in dogs of any breed and at any age. ${ }^{2,-7}$ Posthepatic portal hypertension (eg, right-sided heart failure) never results in APSS formation because pressure in the caudal vena cava is increased along with pressure in the portal vein. ${ }^{2,6}$ A common consequence of any kind of portal hypertension is accumulation of free abdominal fluid; however, portal hypertension cannot be excluded in the absence of ascites. ${ }^{2,8}$

Portosystemic shunting is considered to be congenital if a single or double anomalous vein is present without concurrent portal hypertension. ${ }^{5}$ Congenital portosystemic shunts are classified as intrahepatic or extrahepatic., Intrahepatic CPSSs occur more commonly in large breed dogs and typically result in hepatic encephalopathy before 1 year of age, ${ }^{9,10}$ whereas extrahepatic CPSSs are more common in small breed dogs and may not cause any clinical abnormalities until later in life. ${ }^{5}$ The etiopathogenesis of CPSSs is obscure, except that left-sided intrahepatic CPSSs are believed to represent persistence of the ductus venosus. ${ }^{11,12}$

Less common causes of hyperammonemia in dogs include transient physiologic hyperammonemia in healthy Irish Wolfhound pups ${ }^{13}$ and conditions in which urine that contains ammonia as a result of the activity of urea-splitting microorganisms is absorbed into the systemic circulation. This could happen in dogs with a ruptured urinary bladder or urethra ${ }^{14}$ or after surgical ureterocolonic anastomosis. ${ }^{15}$

Ammonia is 1 of the toxins that plays a role in the development of hepatic encephalopathy. Therefore, detection of a high blood ammonia concentration in a dog can help confirm that CNS abnormalities are a result of hepatic encephalopathy or suggest that nonspecific clinical abnormalities may be related to hyperammonemia. High bile acids concentrations are also suggestive of portosystemic shunting, ${ }^{a}$ but may also be a result of cholestasis and diffuse hepatocellular dysfunction associated with various hepatic disorders. ${ }^{16}$ 
A noninvasive method for differentiating CPSSs from APSSs and urea cycle enzyme deficiency would be helpful clinically because CPSSs require surgical treatment and the other 2 conditions do not. Although ultrasonography is a quick and noninvasive method for imaging the abdominal vessels and organs in unsedated dogs, veterinarians tend to rely on the results of angiographic studies, ${ }^{6,17,18}$ and the few reports ${ }^{12,18-22}$ that have described ultrasonographic findings in dogs with portosystemic shunts have focused only on some aspects of CPSSs without providing detailed anatomic descriptions.

Recently, standard planes for ultrasonographic imaging of the portal system in dogs were described. ${ }^{23}$ The purpose of the study reported here was to determine ultrasonographic abnormalities in dogs with hyperammonemia. Our hypothesis was that ultrasonography could reliably be used to identify CPSSs and APSSs, to identify subtypes of CPSSs, and to rule out portosystemic shunting in dogs with hyperammonemia attributable to other causes.

\section{Criteria for Selection of Cases}

Records of dogs with hyperammonemia that underwent abdominal ultrasonography at the Companion Animal Clinic of Utrecht University between July 2000 and December 2002 were reviewed. Dogs in which ultrasonography had been performed by a single individual (VS) and in which a definitive diagnosis had been made (ie, on the basis of gross findings during laparotomy or necropsy or histologic findings during examination of a liver specimen) or in which characteristic ultrasonographic signs of arterioportal fistulae were identified (ie, an extremely dilated and tortuous portal branch) were included in the study. For this study, dogs were considered to have hyperammonemia if blood ammonia concentration after food had been withheld for 12 hours was greater than the upper limit of the reference range ( 24 to $45 \mu \mathrm{mol} / \mathrm{L}$ ).

\section{Procedures}

Ultrasonography was performed as described. ${ }^{23}$ In general, dogs were not sedated or anesthetized for this procedure. First, B-mode abdominal ultrasonography ${ }^{b}$ was performed, with special attention paid to the presence and amount of free abdominal fluid. The quantity of peritoneal effusion was subjectively estimated and classified as a large (ascites detectable by physical examination), moderate (ascites not detectable by physical examination but easily seen ultrasonographically), or small (thorough ultrasonographic search needed to detect free abdominal fluid) amount. Second, ultrasonographic evaluation of the abdominal vasculature was performed in 7 standard planes. Briefly, with the dog in left lateral recumbency, transverse sections of the portal vein and its branches were obtained via the right intercostal spaces, and longitudinal sections were obtained via the right flank. With the dog in dorsal recumbency, longitudinal sections of the portal vein were obtained via the ventral body wall. With the dog in right lateral recumbency, longitudinal sections were obtained via the left flank to image right gastric-caval shunts and the left testicular or ovarian vein.
In dogs in which the ultrasonographic diagnosis was a CPSS, surgical shunt ligation was recommended unless the dog was $>5$ years old. All ligations were performed by a single individual (FJvS); a standard protocol for shunt ligation was followed. ${ }^{24}$ Only dogs that underwent laparotomy or necropsy were included in the study; dogs that did not undergo surgery because they were $>5$ years old or because the owner declined treatment were excluded.

In dogs in which the ultrasonographic diagnosis was APSSs and portal hypertension, ultrasound-guided core biopsy of the liver was performed and specimens were submitted for histologic examination. Liver biopsy was not performed in dogs with abnormal blood coagulation parameters. Only dogs in which hepatic biopsy specimens were examined histologically were included in the study.

In dogs in which ultrasonography revealed normal abdominal vascular anatomy without any evidence of portal hypertension or anomalous vessels, a rectal ammonia tolerance test was performed as described. ${ }^{25}$ When test results indicated abnormal ammonia metabolism, scintigraphy was performed by injecting technetium Tc $99 \mathrm{~m}$ albumin aggregate into a parenchymal splenic vein under ultrasound guidance, as described. ${ }^{26}$ In addition, ultrasound-guided core biopsy of the liver was performed, and specimens were submitted for histologic examination.

When a dog was examined more than once because of complaints related to hyperammonemia, results of only the first examination were included in the study.

\section{Results}

Ninety dogs met the criteria for inclusion in the study. Most dogs were not sedated or anesthetized during abdominal ultrasonography. However, a litter of 4 puppies were anesthetized during ultrasonography because anesthesia was necessary for collection of blood samples, and 1 dog was anesthetized because ultrasonographic findings were compatible with a leftsided intrahepatic CPSS but the suspected anomalous vein could not be visualized while the dog was awake. The examination was repeated in this dog after it was anesthetized.

In 11 of the 90 dogs, the abdominal vascular anatomy appeared normal ultrasonographically. A CPSS was identified in 61 dogs, and APSSs were identified in 17 dogs, including 3 dogs with arterioportal fistulae and 14 dogs with APSSs attributed to portal hypertension of hepatic origin. In the remaining dog, both an arterioportal fistula and a CPSS were identified.

Dogs with normal abdominal vascular anatomyNo abnormalities were found ultrasonographically in 11 dogs. In 2 of these dogs, repeated measurements of blood ammonia concentrations and results of a rectal ammonia tolerance test were normal.

In the remaining 9 dogs, representing puppies from 3 litters, results of rectal ammonia tolerance tests were abnormal, serum bile acids concentrations were normal, scintigraphy excluded portosystemic shunting, ultrasonography revealed a small amount of free 
abdominal fluid, and histologic examination of hepatic biopsy specimens did not reveal any abnormalities. These 9 dogs consisted of a litter of 4 Belgian Malinois puppies, a litter of 2 Cairn Terrier puppies, and 3 Irish Wolfhound puppies from a litter of 4 (the fourth Irish Wolfhound puppy from this litter had a left-sided intrahepatic CPSS). Puppies were 48, 59, and 63 days old. Metabolic examination of blood and liver samples from the 3 Irish Wolfhound puppies revealed argininosuccinate synthetase enzyme deficiency.

Congenital portosystemic shunts-Congenital portosystemic shunt was the ultrasonographic diagnosis in 61 dogs. The liver was always small, sometimes to such an extent that parts of the stomach were in direct contact with the diaphragm. Free abdominal fluid was seen only in young puppies and only in small amounts.

Congenital portosystemic shunts were categorized as intrahepatic or extrahepatic on the basis of origin of the shunt. Intrahepatic portosystemic shunts were identified in 19 dogs. Mean age of the dogs at the time of surgery was 5.9 months (range, 2.5 to 17.6 months). All dogs were large breed dogs, except for a Border Collie. Intrahepatic CPSSs originated from the left (14 dogs) or right (5) portal branch and appeared to be a direct continuation of the portal vein, as diameters of the shunt and affected portal branch were the same as the diameter of the portal vein. All intrahepatic CPSSs terminated in the caudal vena cava either directly or via a hepatic vein. The intrahepatic CPSS was a single vessel in 18 dogs and consisted of 2 vessels in 1 dog.

Intrahepatic CPSSs that originated from the left portal branch always ran cranioventrally and to the left (as expected for a normal left portal branch) until the level of the diaphragm, then turned abruptly dorsally to enter the caudal vena cava via a dilated segment of the left hepatic vein. In these dogs, the right portal branch could never be visualized. A thin vessel with fast hepatopetal flow that was found at the place where the right portal branch was expected appeared to be a hepatic arterial branch when examined by means of pulsed-wave Doppler ultrasonography.

Intrahepatic CPSSs that originated from the right portal branch had a segment that consistently ran dorsolaterally and to the right from the portal vein (as expected for a normal right portal branch), but then instead of ramifying, turned medially to enter the caudal vena cava (Fig 1). This dorsolaterally directed segment was short in 2 dogs and long in 3 . One of the long shunts was double; this was identified in the Border Collie. The left portal branch could never be visualized in dogs with intrahepatic CPSSs originating from the right portal branch.

Extrahepatic portosystemic shunts were identified in 42 dogs. Mean age of these dogs at the time of surgery was 16 months (range, 2.9 to 66.4 months). All dogs were small breed dogs except for a Labrador Retriever. Extrahepatic CPSSs originated from the splenic vein, from the right gastric vein, or simultaneously from both and entered the abdominal portion of the caudal vena cava or the thorax. Extrahepatic CPSSs were divided into 4 groups on the basis of their origins and terminations: 24 dogs had a splenic-caval, 9 had a right gastriccaval, 8 had a splenic-azygos, and 1 had a right gastric- azygos shunt. Dogs with 2 shunt loops (1 from the right gastric vein and the other from the splenic vein) were classified as having right gastric-caval shunts because this was the dominant loop. In all dogs, ultrasonographic findings were in agreement with surgical findings.

The most common type of congenital CPSS was a splenic-caval shunt. This usually formed a short single loop between the portal vein and caudal vena cava (Fig 2). Although in 2 dogs the anomalous vein had a long cranial loop, the points of origin and termination

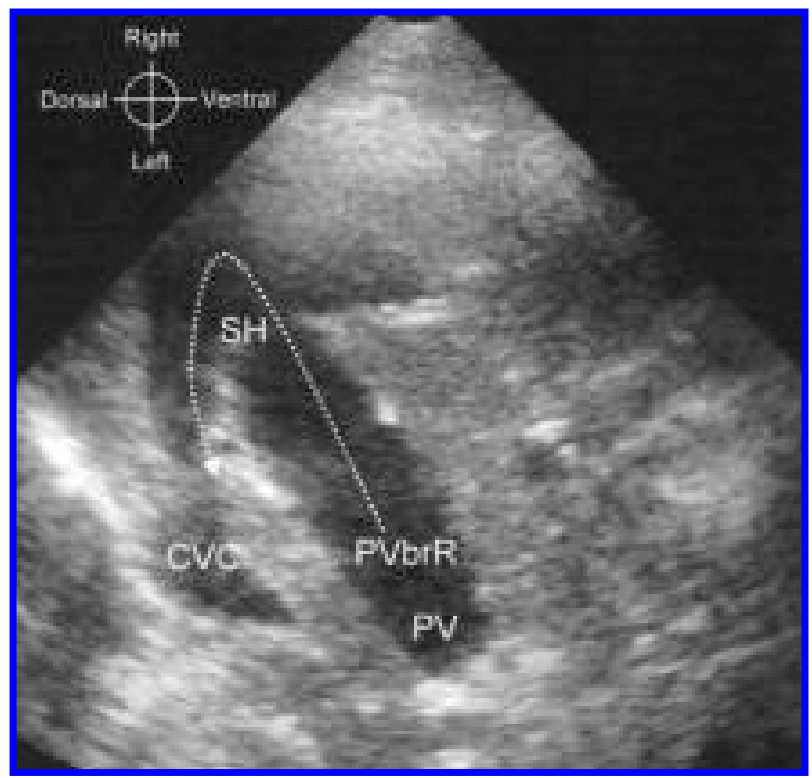

Figure 1-Ultrasonogram of a 5.5-month-old male Labrador Retriever with an intrahepatic congenital portosystemic shunt $(\mathrm{SH})$ arising from the right portal branch (PVbrR) of the portal vein (PV). Note that the PVbrR is as wide as the PV and remains wide towards the periphery. The direct connection between the PV and caudal vena cava (CVC) can be appreciated in this single image.

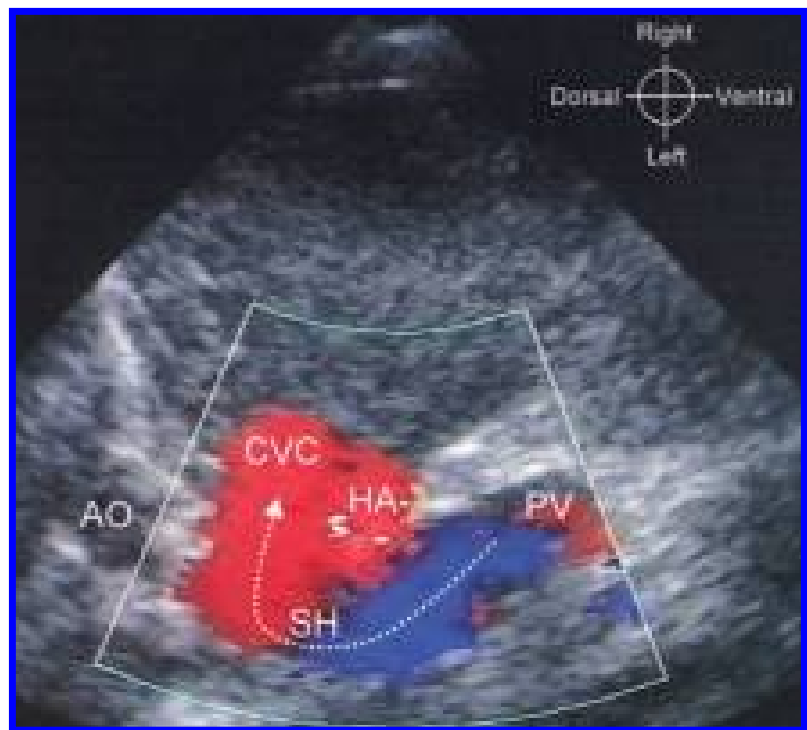

Figure 2-Color flow Doppler ultrasonogram of a 3.5-month-old female Jack Russell Terrier with a congenital extrahepatic splenic-caval shunt. Cross sections of the aorta (AO), CVC, and $\mathrm{PV}$ are seen. Between the CVC and the PV, the hepatic artery (HA) is seen. A short anomalous shunt ( $\mathrm{SH}$ ) makes a direct connection between the PV and CVC. The direction of blood flow in the $\mathrm{SH}$ is from the PV towards the CVC (dotted arrow). See Figure 1 for remainder of key. 
were the same as in dogs with short loops. Spleniccaval CPSSs originated from the splenic vein. However, because the point of origin was close to the point where the splenic vein entered the portal vein and the short segment of the splenic vein that was between the portal vein and the origin of the shunt was dilated and had hepatofugal blood flow, the CPSS seemed ultrasonographically to originate from the portal vein itself and the splenic vein seemed to enter the shunting vessel (Fig 3). The origin of all spleniccaval shunts was slightly cranial to the level where the celiac artery originated from the aorta. The termination of the splenic-caval shunts in the caudal vena cava was always at the same point, slightly cranial to the level of shunt origin (Fig 4).

In dogs with splenic-azygos shunts, the point of
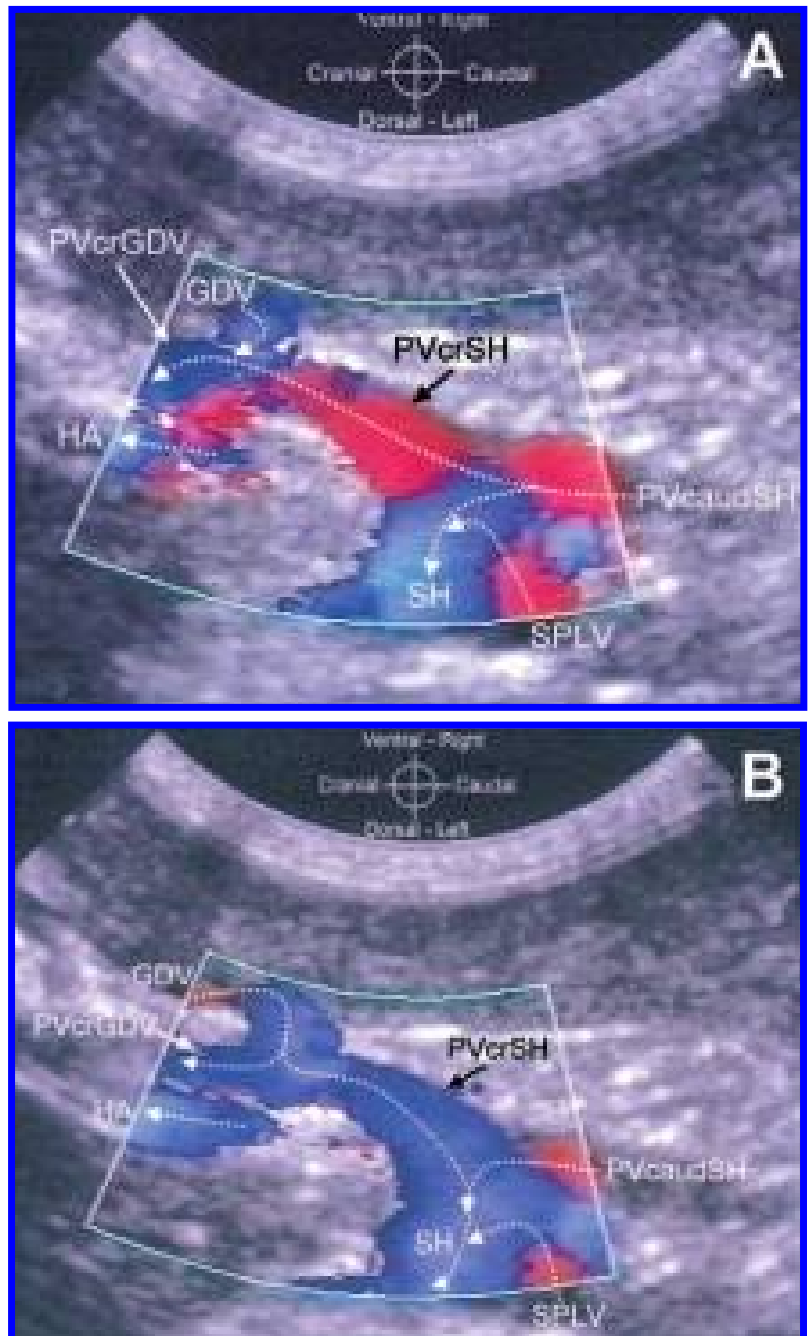

Figure 3-Color flow Doppler ultrasonograms of a 4-month-old male Yorkshire Terrier with a congenital extrahepatic spleniccaval shunt (SH). Images were obtained 2 seconds apart and indicate that blood flow in the PV segment between the SH origin and the gastroduodenal vein ( $\mathrm{PVCrSH}$ ) had a to-and-fro pattern (ie, alternating hepatopetal $[A]$ and hepatofugal $[B]$ flow). Continuous hepatopetal flow is seen in the PV segment cranial to the entering point of the gastroduodenal vein (PVcrGDV) and in the PV segment caudal to the SH origin (PVcaudSH). Dotted arrows indicate the direction of blood flow. $\mathrm{HA}=$ Hepatic artery. $\mathrm{SPLV}=$ Splenic vein. GDV = Gastroduodenal vein. See Figure for remainder of key. origin was the same as described for dogs with spleniccaval shunts. The shunt vessel ran toward the caudal vena cava, but instead of entering it at the point where splenic-caval shunts terminated, ran further dorsal to the caudal vena cava and eventually entered the thorax (Fig 4).

Shunts that originated from the splenic vein always had a diameter greater than that of the portal vein caudal to the shunt origin (Fig 3). Diameter of the portal vein segment cranial to the shunt origin was less than the diameter of the portal vein segment caudal to the shunt origin in all 32 dogs with shunts originating from the splenic vein. In some dogs, the portal vein segment cranial to the shunt origin was so thin that it could not be visualized ultrasonographically. The direction of blood flow in the portal vein segment cra-
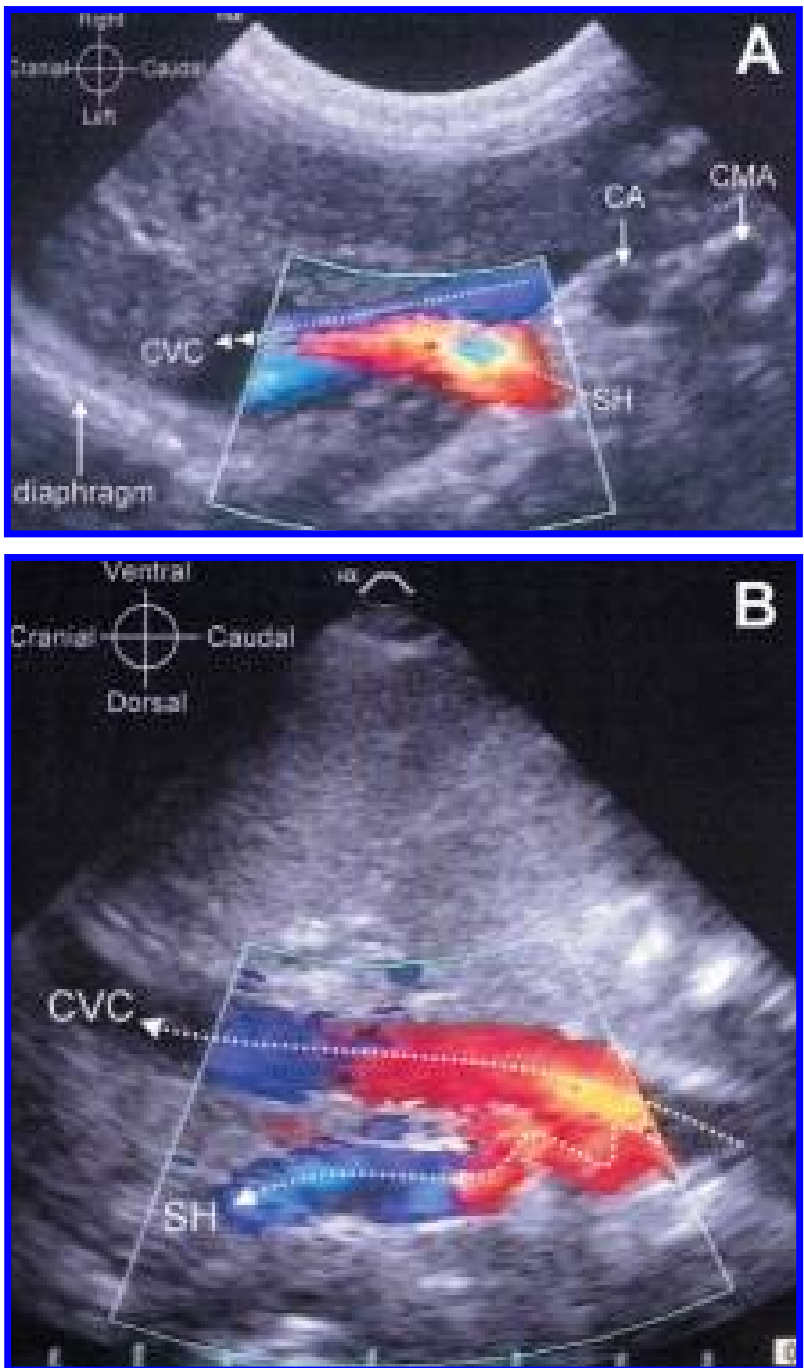

Figure 4-Color flow Doppler ultrasonograms of 2 dogs with extrahepatic congenital portosystemic shunts. A-Image obtained from a 3-month-old female Cairn Terrier with a spleniccaval shunt. Notice that the shunt (SH) enters the CVC slightly cranial to the point where the celiac artery (CA) originates from the aorta. Dotted arrows indicate the direction of blood flow. $\mathrm{CMA}=$ Cranial mesenteric artery. B-Image obtained from a 3.5-month-old male Jack Russell Terrier with a splenic-azygos shunt. The shunt runs dorsal to the CVC and enters the thorax. Dotted arrows indicate the direction of blood flow. See Figure 1 for remainder of key. 
nial to the shunt origin was hepatofugal or to-and-fro in 17 of the 32 dogs, as determined by means of color flow Doppler ultrasonography. In the remaining dogs, this segment could not be visualized or the flow was normal (ie, hepatopetal). The portal vein segment cranial to the origin of the gastroduodenal vein could only occasionally be imaged. Whenever this portal vein segment was visualized, its flow was always hepatopetal, even if hepatofugal blood flow was seen in the segment cranial to the shunt origin.

Most of the 9 dogs with right gastric-caval shunts had double shunts consisting of a cranial and a caudal shunt loop; the 2 loops anastomosed before entering the systemic venous system. The cranial loop arose from the right gastric vein; the morphology of the shunt origin was slightly variable depending on whether the right gastric vein was a tributary of the gastroduodenal vein or of the portal vein itself. In both cases, the shunt (ie, the dilated right gastric vein) originated immediately caudal to the portal bifurcation. When the right gastric vein was a tributary of the gastroduodenal vein, the origin of the shunt (ie, the right gastric vein) was very close to the point where the gastroduodenal vein entered the portal vein, and the short segment of the gastroduodenal vein that was between the portal vein and the origin of the right gastric vein was dilated and had hepatofugal blood flow. Regardless of the anatomic variation of the right gastric vein, the course of the shunt was always the same, in that it made a long loop from the liver hilus, running lateral to the left body wall, then turned caudomedially and entered the caudal vena cava at the point where splenic-caval shunts terminated (ie, slightly cranial to the level where the celiac artery originated from the aorta; Fig 5). In 1 dog, the shunt did not enter the caudal vena cava at this point, but ran dorsal to the caudal vena cava and entered the thorax. In this dog, the ultrasonographic diagnosis was right gastric-azygos shunt. All CPSSs that arose from the right gastric vein were wide, with a diameter comparable to that of the caudal vena cava. At surgery, the cranial loop of right gastriccaval shunts was found to follow the lesser curvature of the stomach, similarly to the normal right gastric vein. The caudal shunt loop, which was occasionally absent, originated at the region where splenic-caval shunts would be expected, but unlike splenic-caval CPSSs, the caudal loop of right gastric-caval shunts ran from caudal to cranial and not from ventral to dorsal like splenic-caval shunts. Moreover, the caudal loop formed a longer shunt loop than did the splenic-caval shunts. The caudal loop drained blood from the portal vein via a dilated segment of the splenic vein, through the left gastric vein to the right gastric vein. Because the cranial shunt loop (right gastric vein to caudal vena cava) and the caudal shunt loop (splenic vein to left gastric vein to right gastric vein) anastomosed, a common trunk entered the caudal vena cava. The portal vein was slightly thinner cranial to the origin of the caudal shunt loop (ie, cranial to the splenic vein), and the direction of blood flow in this segment was always hepatopetal. The portal vein cranial to the origin of the cranial shunt loop was so thin that it could never be visualized ultrasonographically.
Acquired portosystemic shunts-Portal hypertension with APSSs was the ultrasonographic diagnosis in 17 dogs. In all of these dogs, a dilated left testicular or ovarian vein was seen. This vein ran parallel to the caudal vena cava and entered the mid-portion of the left renal vein from caudally (Fig 6). The diameter of the testicular or ovarian vein was usually similar to that of the renal vein, but in some cases it was much greater, and the vein had a straight or tortuous course. Many small tortuous veins were often observed around the left renal vein (Fig 7). In $1 \mathrm{dog}$, the dilated left ovarian vein could be followed caudally to a conglomeration of smaller vessels, whereas in another dog, it could be followed to its origin from the splenic vein at the splenic hilus. In the remaining dogs, the dilated left testicular or ovarian vein could not be followed.

In addition to the dilated left testicular or ovarian vein, the origin of an APSS from the portal vein was seen in 4 dogs at the point where congenital extrahepatic splenic-caval shunts would be expected

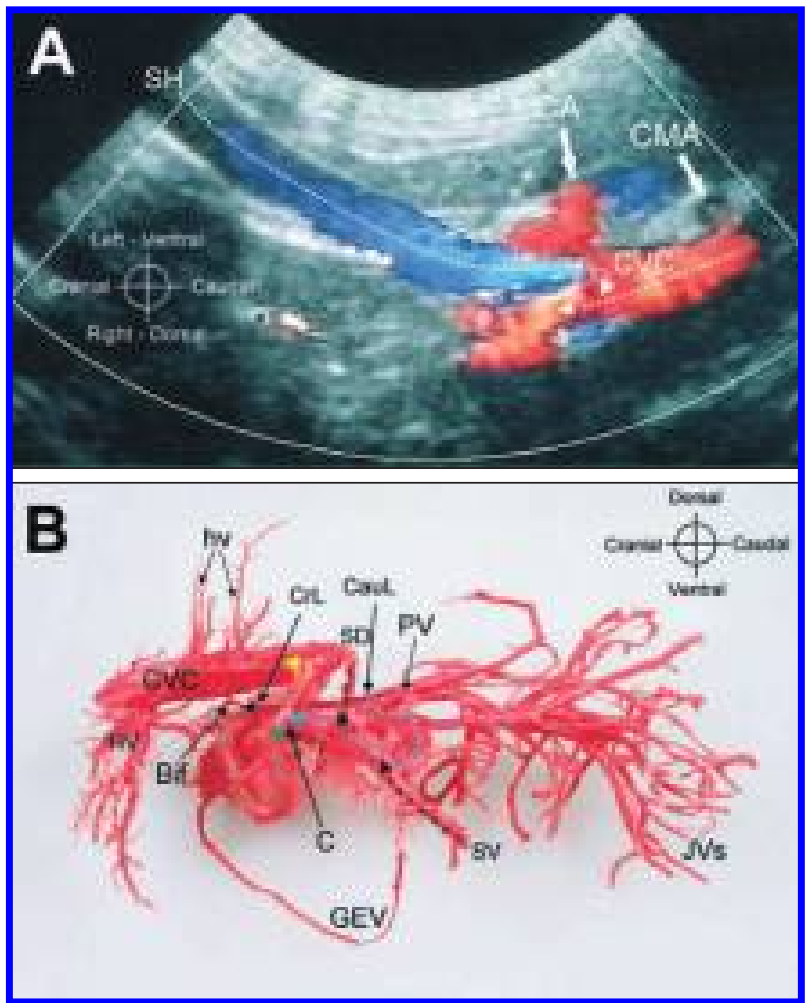

Figure 5-Congenital extrahepatic right gastric-caval shunt. AColor Doppler ultrasonogram of the cranial shunt loop in a 6.5month-old female Yorkshire Terrier imaged via the left abdominal wall with the dog in right lateral recumbency. Note the large-caliber shunting vessel (SH) immediately under the left body wall. The shunt terminates in the CVC slightly cranial to the point where the CA originates from the AO. Dotted arrows indicate the direction of blood flow. CMA = Cranial mesenteric artery. BCorrosion cast of the abdominal vasculature of a Yorkshire Terrier with a right gastric-caval shunt. The cranial shunt loop (green arrow) and the caudal shunt loop (blue arrow) anastomose with each other (C), and the shunt vessel (yellow arrow) enters the CVC. The PV becomes narrow cranial to the origin of the caudal shunt loop (CauL) and even more narrow cranial to the origin of the cranial shunt loop (CrL). Arrows indicate direction of blood flow. Bif = Portal bifurcation. HV = Left hepatic vein. hv = Hepatic veins. JVs = Jejunal veins. GEV = Right and left gastroepiploic veins. $S V=$ Ventral branch of the splenic vein. $S D=$ Dorsal branch of the splenic vein. See Figures 1 and 2 for remainder of key. 


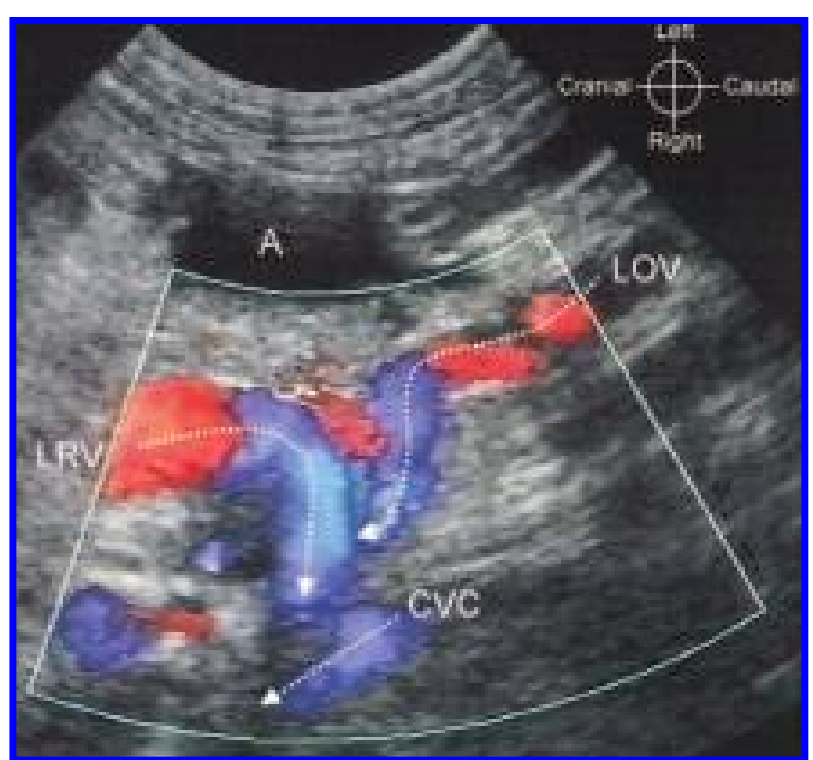

Figure 6-Color flow Doppler ultrasonogram of a dilated left ovarian vein (LOV) in a 6.5-year-old Jack Russell Terrier with acquired portosystemic shunts (splenic-renal collateral vessels) secondary to primary hypoplasia of the PV. Dotted arrows indicate the direction of blood flow. $A=$ Ascitic fluid. $L R V=$ Left renal vein. See Figure 1 for remainder of key.

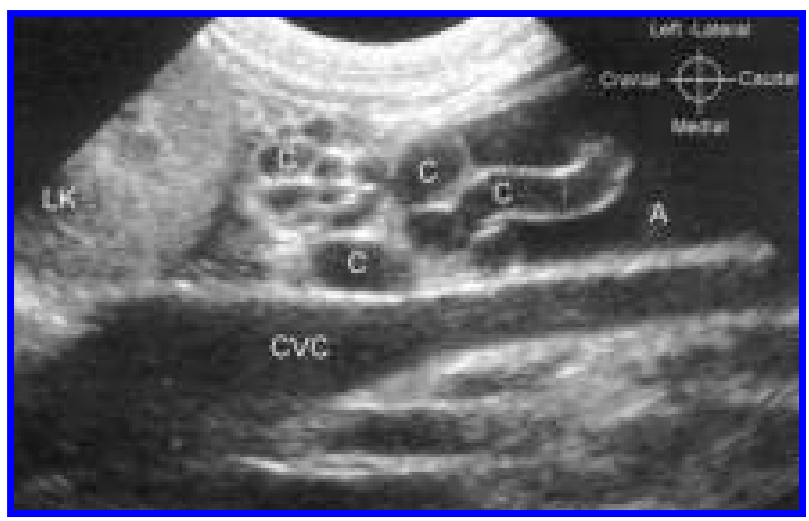

Figure 7-Ultrasonogram of a 1-year-old female Dutch Schapendoes with acquired portosystemic shunts secondary to sustained portal hypertension of hepatic origin. Collateral vessels (C) can be seen caudal to the left kidney (LK). A = Ascitic fluid. See Figure 1 for remainder of key.

to arise (Fig 8A). These APSSs originated directly from the portal vein, and their relation to the splenic vein could not be revealed. In 2 of the 4 dogs, blood flow in the entire portal vein was so slow that no color signals were detected with the lowest possible pulse repetition frequency setting. In the other 2 dogs, the velocity of blood flow caudal to the origin of the APSS was estimated to be normal with color Doppler ultrasonography; however, blood flow in the portal vein cranial to the origin of the APSS was hepatofugal (Fig 8).

The morphology of APSSs that originated from the portal vein was similar to that of congenital extrahepatic splenic-caval shunts. Moreover, hepatofugal blood flow could be seen in the portal vein cranial to the origin of the anomalous vein. The major difference was that APSSs ran caudally from the point of origin and tended to disappear among the intestines.
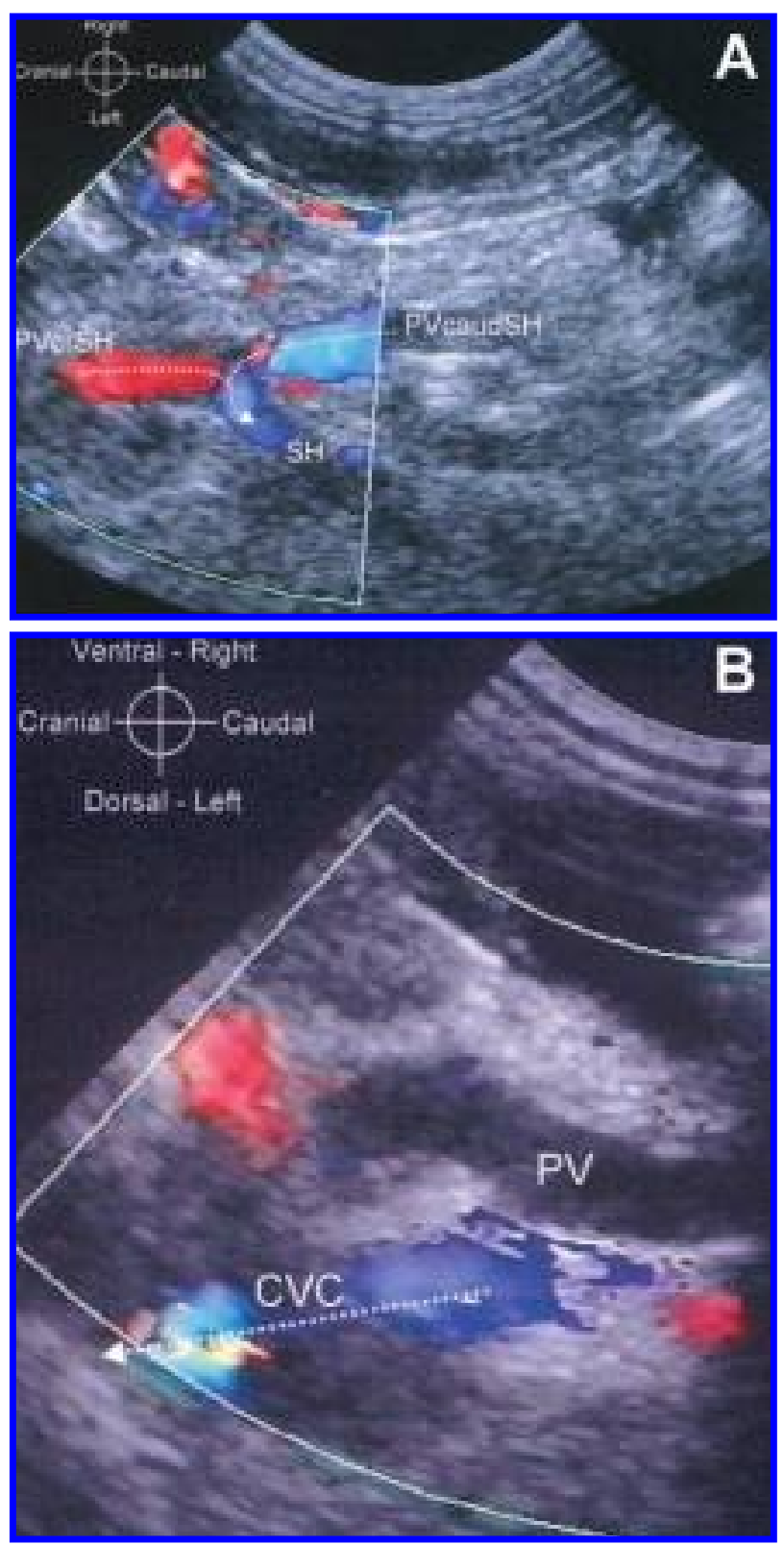

Figure 8-Color flow Doppler ultrasonograms of 2 dogs with acquired portosystemic shunts. A-Image obtained from a 5year-old West Highland White Terrier with sustained portal hypertension of hepatic origin. Blood flow in the PV cranial to the origin of the shunt (PVCrSH) is hepatofugal, and the shunt (SH) runs caudally. Dotted arrows indicate the direction of blood flow. PVcaudSH = Portal vein caudal to the shunt origin. B-Image from a 6.5-year-old female Jack Russell Terrier with sustained portal hypertension secondary to primary hypoplasia of the portal vein. Blood flow is not detectable in the PV, whereas aliasing artifact is seen in the CVC. Dotted arrow indicates the direction of blood flow. See Figure 1 for remainder of key.

Furthermore, the diameters of the portal vein cranial and caudal to the origin of the APSS were approximately equal. In contrast, congenital extrahepatic splenic-caval or splenic-azygos shunts ran cranially from their origin and could always be followed to their termination in the caudal vena cava or to the point where they entered the thorax. Furthermore, the portal vein segment that was cranial to the origin 
of a splenic-caval or splenic-azygos shunt was always thinner than the portal vein segment caudal to it. Moreover, an APSS was never wider than the portal vein caudal to the anomalous vein, whereas a CPSS was usually wider. In addition to these features, simultaneous detection of a dilated left testicular or ovarian vein always proved that an extrahepatic anomalous vein that originated from the portal vein was an APSS.

Three underlying diseases were associated with detection of APSSs: arterioportal fistulae, primary hypoplasia of the portal vein (PHPV), and chronic hepatitis. Arterioportal fistulae were diagnosed ultrasonographically, whereas chronic hepatitis and PHPV were differentiated by histologic examination of liver biopsy specimens. The size of the spleen was normal in all dogs with portal hypertension.

Arterioportal fistulae were identified in 4 dogs; however, the classic ultrasonographic appearance (ie, ascites, an extremely dilated and tortuous portal branch in a liver lobe, hepatofugal blood flow in the portal vein, and APSSs; Fig 9) was seen only in 2, including a 6-month-old American Staffordshire Terrier and a 2.5-month-old Bullmastiff. A 3-year-old Basset Hound was examined that did not have any free abdominal fluid, but in which the other 3 features were found. A 9-month-old Labrador Retriever was examined that had only an extremely dilated and tortuous portal branch without ascites or APSSs, and the blood flow in the portal vein was normal (ie, hepatopetal). These findings suggested that in addition to the arterioportal communication, an intrahepatic portocaval communication had to exist, which was confirmed during necropsy. Histologic examination of liver biopsy specimens from the Labrador Retriever and Basset Hound revealed hypoplastic portal branches.

Primary hypoplasia of the portal vein was identified as the cause of APSSs in 7 dogs (6 females and 1 male) by means of histologic examination of liver biop-

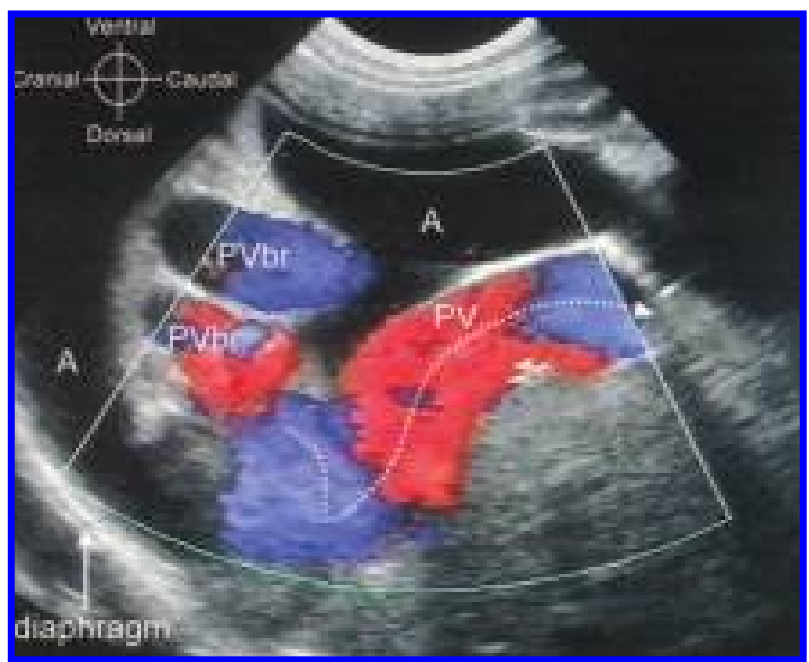

Figure 9-Color flow Doppler ultrasonogram of a 6-month-old male American Staffordshire Terrier with a congenital arterioportal fistula. Notice the dilated portal branches (PVbr) in the affected liver lobe. Direction of blood flow in the PV is hepatofugal. Dotted arrow indicates the direction of blood flow. $\mathrm{A}=$ Ascitic fluid. See Figure 1 for remainder of key. sy specimens. Mean age of these dogs was 3.4 years (range, 0.5 to 6.5 years). The liver was small in all dogs and had a normal (2 dogs) or slightly irregular echoic structure ( 5 dogs). Ascites was found at physical examination only in $1 \mathrm{dog}$. In 3 other dogs, a moderate amount of free abdominal fluid was detected ultrasonographically, and in the remaining 3 dogs, no free abdominal fluid was seen.

Chronic hepatitis with or without cirrhosis was the histologic diagnosis in 7 dogs $(3$ females and 4 males). Mean age of the dogs was 5.5 years (range, 2.9 to 9.5 years). Six of the 7 dogs had severe ascites at physical examination and irregular hepatic structure detected ultrasonographically. One dog had only a moderate amount of free abdominal fluid and an ultrasonographically normal liver.

Double caudal vena cava-Double caudal vena cava was found in 3 dogs with an extrahepatic CPSS. In dogs with double caudal vena cava, the left and right common iliac veins fused to form the caudal vena cava more cranially than normal, between the left and right renal veins. Hence, the left renal vein entered the left common iliac vein, and the right renal vein entered the caudal vena cava. The left and right common iliac veins had the same diameters and ran on either side of the aorta (Fig 10).
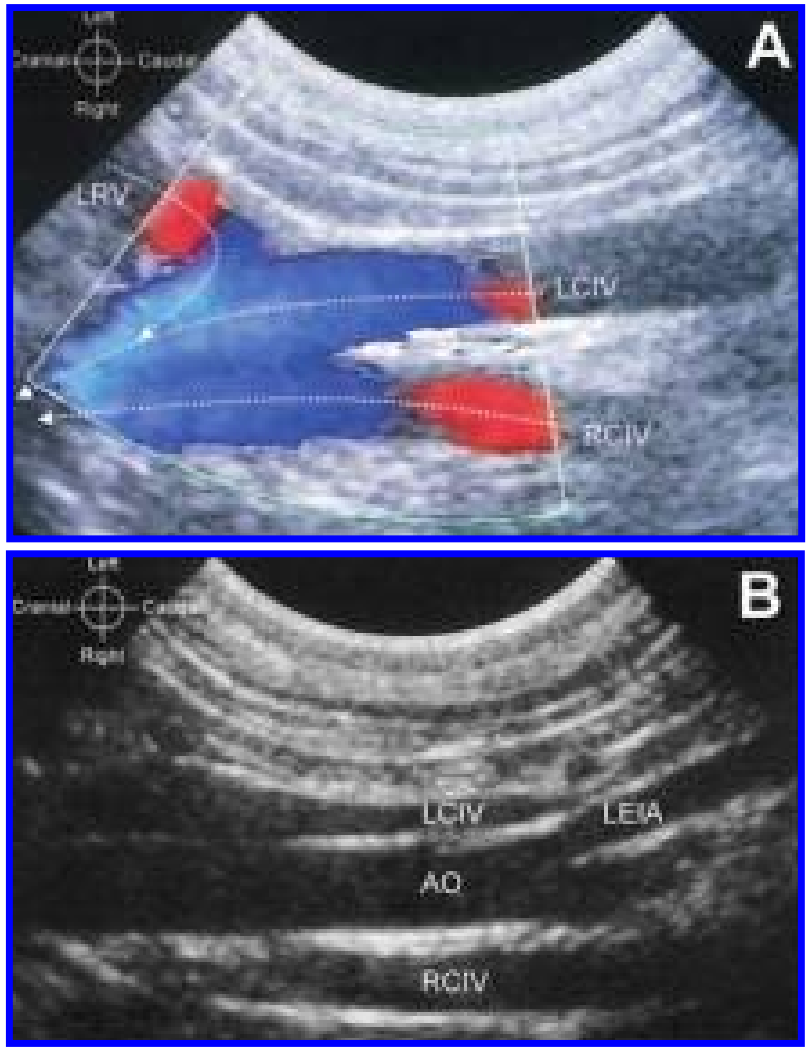

Figure 10-Ultrasonograms of a 3-month-old female Cairn Terrier with a double CVC. A-Color flow Doppler ultrasonogram of the LRV as it enters the left common iliac vein (LCIV). Dotted arrows indicate the direction of blood flow. RCIV = Right common iliac vein. B-Gray scale image of the aortic trifurcation. The left and right common iliac veins can be seen on either side of the $\mathrm{AO}$ beyond the level of the aortic trifurcation. LEIA = Left external iliac artery. See Figures 1, 2, and 6 for remainder of key. 


\section{Discussion}

Dogs in the present study with hyperammonemia were identified as having 1 of the following diseases: CPSS, APSSs secondary to PHPV, APSSs secondary to arterioportal fistulae, APSSs secondary to chronic hepatitis, and urea cycle enzyme deficiency. However, the incidence of APSSs and CPSSs in the present study does not represent the incidence of these disorders in the clinic's population because dogs with an ultrasonographic diagnosis of APSSs or with congenital extrahepatic splenic-azygos shunts were more often excluded from the study population than were dogs with intrahepatic CPSSs. For instance, dogs suspected to have APSSs were excluded if coagulation times were sufficiently prolonged that liver biopsy could not be safely performed. Hence, the ultrasonographic diagnosis remained unconfirmed. Similarly, in dogs suspected to have CPSSs that were $>5$ years old, dietary and medical management was recommended instead of surgical shunt ligation. Therefore, several dogs with an ultrasonographic diagnosis of congenital extrahepatic splenic-azygos shunt were not included in the study population because the ultrasonographic diagnosis was not confirmed surgically.

Two basic types of intrahepatic CPSSs and 4 basic types of extrahepatic CPSSs were found in this study. These categories were established on the basis of the origin and termination of the anomalous veins. ${ }^{10}$ Intrahepatic CPSSs were categorized as left division shunts (ie, shunts originating from the left portal branch), right division shunts (ie, long shunts originating from the right portal branch), and central division shunts (ie, short shunts originating from the right portal branch). These categories are in agreement with results of angiographic studies. ${ }^{12}$

Previous descriptions of extrahepatic CPSSs used the term "portocaval." ${ }^{18,19}$ Indeed, on portographic and ultrasonographic images, all extrahepatic CPSSs seem to arise from the portal vein itself and divert blood from the portal vein. However, necropsy studies ${ }^{10,20}$ showed that they actually originate either from the splenic vein or from the right gastric vein. Ultrasonographically, we confirmed these findings and found that all extrahepatic CPSSs originated very close to the point where the splenic vein (in dogs with splenic-caval or splenic-azygos shunts) or the right gastric vein (in dogs with right gastric-caval shunts) entered the portal vein, and the short segment between the portal vein and the shunt origin was always dilated, with hepatofugal blood flow. What actually happens in dogs with extrahepatic CPSSs originating from the splenic vein is that the shunt drains blood from the portal and splenic veins to the caudal vena cava or azygos vein. In dogs in which blood flow in the portal vein segment that is cranial to the shunt origin is hepatofugal, blood from the gastroduodenal vein flows via the portal vein to the shunt as well.

In dogs with extrahepatic CPSSs originating from the right gastric vein, there were 2 anatomic variations regarding the cranial loop of the shunt. ${ }^{27}$ In some dogs, the right gastric vein is a tributary of the portal vein and is located between the portal bifurcation and the gastroduodenal vein. In others, the right gastric vein is a tributary of the gastroduodenal vein. When the right gastric vein is a direct tributary of the portal vein, blood from the portal vein is drained via the right gastric vein to the caudal vena cava or azygos vein. When the right gastric vein is a tributary of the gastroduodenal vein, blood from the portal vein is drained via a short and dilated segment of the gastroduodenal vein through the right gastric vein into the caudal vena cava or azygos vein, and blood from the gastroduodenal vein flows through the shunt (ie, the right gastric vein) without reaching the portal vein. The caudal shunt loop of a right gastric-caval shunt actually functions similarly to a splenic-caval shunt, except for the fact that blood flow is always hepatopetal in the portal vein segment cranial to the origin of the caudal loop (ie, between the origins of the 2 shunt loops).

In dogs in the present study with extrahepatic CPSSs that entered the thorax, we assumed that the shunt terminated in the azygos vein, but we could not visualize this segment ultrasonographically because of the air-filled lungs.

Hepatofugal portal flow has been reported in association with extrahepatic CPSS in 1 dog ${ }^{18}$ but no explanation was given. Hepatofugal blood flow in the portal vein cranial to the shunt origin was observed in dogs in the present study with APSSs and with congenital extrahepatic splenic-caval and splenic-azygos shunts. However, the cause of hepatofugal blood flow is probably different. In dogs with CPSSs, blood from the gastroduodenal vein was found to be responsible for the hepatofugal flow because the shunt had a lower resistance than the hepatic sinusoids. This was confirmed by detection of hepatopetal blood flow in the portal vein cranial to the point where the gastroduodenal vein entered. Preoperative detection of the direction of blood flow in the portal vein segment cranial to the origin of a CPSS may be important in determining the degree of portal vein hypoplasia, hence facilitating prediction of surgical outcome. ${ }^{28}$ In dogs with hepatopetal or to-and-fro blood flow, an excellent prognosis could be expected because the direction of blood flow is continuously or at least intermittently physiologic, whereas in dogs with hepatofugal flow, the surgical prognosis can range from poor to excellent. In human patients with hepatic cirrhosis, microscopic intrahepatic arterioportal communications were found to be responsible for hepatofugal blood flow and were thought to have been formed because of the disorganized hepatic architecture. ${ }^{29,30}$ This might also be the case in $\operatorname{dog} 5^{5,31}$; however, visualizing the portal branches in dogs with portal hypertension to determine whether blood flow was indeed hepatofugal was impossible in any of the dogs in our study. Intraoperative Doppler ultrasonography of the portal branches ${ }^{32}$ or angiography by superselective catheterization of a hepatic branch of the hepatic artery would be necessary to reveal whether intrahepatic arterioportal communications are indeed responsible for hepatofugal blood flow in dogs with sustained portal hypertension.

Primary hypoplasia of the portal vein is a distinct congenital disorder characterized by hypoplasia of the intrahepatic portal branches and sometimes also of the portal vein. ${ }^{4}$ In mild cases, portal hypertension and 
APSSs do not develop; hence, blood ammonia concentration remains normal. However, in severe cases, APSSs develop as a consequence of sustained hepatic portal hypertension. The severe form of PHPV has also been described as noncirrhotic portal hypertension. ${ }^{6,33-35}$ Hepatic microvascular dysplasia was described as a condition that results in high serum bile acids concentrations without demonstrable macroscopic portosystemic shunting and was speculated to cause hepatic encephalopathy because of suspected microscopic intrahepatic portosystemic communications. ${ }^{36-39}$ However, blood ammonia concentrations in these dogs have never been determined. Because the histologic changes in the liver, as well as the clinical and laboratory findings in these dogs, were identical with findings in dogs with the mild form of PHPV, the World Small Animal Veterinary Association's liver diseases and pathology standardization research group agreed that the described condition is actually PHPV. ${ }^{35, c}$

The etiopathogenesis of secondary portal vein hypoplasia is different from that of PHPV. Secondary hypoplasia of the portal branches can be found when the blood supply of the liver from the portal vein is reduced as, for instance, with portal vein thrombosis or CPSS. Because most CPSSs have a diameter equal to or larger than the diameter of the portal vein segment caudal to the shunt, they offer a lower resistance route to the systemic circulation. Therefore, the portal vein cranial to the shunt origin and the intrahepatic portal branches are hypoperfused.

Ultrasonographically, secondary portal vein hypoplasia is usually recognizable in dogs with intrahepatic CPSSs, in which the nonaffected portal branch is frequently unrecognizably thin, and in dogs with extrahepatic CPSSs, in which the portal vein segment that is cranial to the shunt origin is much thinner than the segment that is caudal to it. In most dogs with extrahepatic CPSSs, the left and right portal branches are also very thin. At the microscopic level, the smallest portal branches are also thinner than normal; however, at this level, secondary portal vein hypoplasia is histologically indistinguishable from PHPV., ${ }^{4,5}$ The situation can be more complicated because PHPV can develop alone or in conjunction with arterioportal fistulae ${ }^{40}$ or CPSSs. When a CPSS and PHPV are present simultaneously in a dog, PHPV cannot be diagnosed preoperatively. In these dogs, portal hypertension and APSSs will not develop because there is already an existing connection between the portal and the systemic veins in the form of a CPSS. Currently, the earliest time when PHPV can be suspected in a dog that has a simultaneous CPSS is during surgical attenuation of the CPSS. $^{28}$

Congenital arterioportal fistulae are single or multiple connections of a portal branch and a hepatic arterial branch that result in portal hypertension and formation of APSSs. Ultrasonographically, the presence of an extremely dilated and tortuous portal branch in a liver lobe is a pathognomic finding. ${ }^{7}$ The high arterial pressure causes dilation of the affected portal branch and hepatofugal blood flow in the affected portal branch and portal vein. Similarly to a reported case, ${ }^{40}$ both dogs in the present study with arterioportal fistu- lae that underwent liver biopsy also had PHPV. Surgical resection of the affected liver lobes should only be considered when PHPV is excluded by histologic examination of liver biopsy specimens because after the arterioportal communication is surgically closed, portal blood will continue to flow towards the lower resistance (ie, the APSSs) and not towards the hypoplastic portal branches.

The development of portal hypertension and APSSs in dogs with cirrhosis is well documented and associated with disorganized hepatic architecture secondary to formation of connective tissue and regenerative nodules. ${ }^{2,8}$ Portal hypertension in dogs with chronic hepatitis develops as a result of intrahepatic inflammatory and fibrotic changes. ${ }^{2,41}$ As a result of these pathologic processes, portal branches and hepatic veins in the liver lobes are compressed and portal hypertension develops.

Because no studies of the ultrasonographic diagnosis of APSSs have been published previously, the ultrasonographic features of APSSs have not been described in detail yet. Measuring portal flow velocity has been suggested as a way to differentiate APSSs from CPSSs $^{18,42}$ because ultrasonographic signs of APSSs were thought to be variable. ${ }^{42}$ However, we found that slow portal blood flow is not necessarily present in all dogs with portal hypertension. In contrast, a dilated left testicular or ovarian vein was found to be a reliable indicator of APSSs. The dilated left testicular or ovarian vein is the result of splenic-renal collateral circulation, which is the most consistently observed route of APSSs in dogs. ${ }^{3,4,31}$ The left testicular or ovarian vein enters the left renal vein in normal dogs, but is so thin that it cannot be visualized ultrasonographically. As a result of sustained hepatic or prehepatic portal hypertension, the virtual communications between the velaromental radicles of the splenic vein and the mesocolic radicles of the left testicular or ovarian vein become functional and these rudimentary vessels dilate, allowing portal blood to shunt into the systemic veins and resulting in dilation of the left testicular or ovarian vein. ${ }^{3}$ Noninvasive diagnosis of APSSs is essential because patients with portal hypertension and hyperammonemia have a high risk of anesthetic complications. ${ }^{43}$ Furthermore, there is nothing in the abdominal cavity that could surgically be corrected in these dogs except in some rare instances, such as dogs with circumscribed stenosis of the portal vein. ${ }^{44}$ Surgical banding of the caudal vena cava has been described to reduce portosystemic shunting via APSSs; however, this method did not bring satisfactory results ${ }^{45}$ and, hence, is not recommended.

In dogs with splenic-renal collateral vessels, a relatively wide and straight collateral vein can regularly be seen. This vein should not be mistaken for a CPSS. We believe that conditions that were described as splenic-mesenteric-renal shunt in $2 \operatorname{dogs}^{46}$ and as suspected microscopic hepatic arteriovenous fistulae ${ }^{16}$ were both instances of PHPV with acquired splenicrenal collateral vessels.

A double caudal vena cava is a clinically unimportant congenital vascular anomaly. ${ }^{47}$ To our knowledge, ultrasonographic diagnosis of this condition has not 
been reported previously. The only clinical significance of a double caudal vena cava is that the left common iliac vein may be mistaken for a dilated left testicular or ovarian vein. If a vein is found on the left side of the aorta caudal to the left kidney and running parallel to the caudal vena cava, thorough evaluation of this vein is necessary to decide whether the dog has a double caudal vena cava or APSSs. The left and right common iliac veins in dogs with double caudal vena cava have the same diameter and run symmetrically on either side of the aorta, whereas a dilated left testicular or ovarian vein may be tortuous, and its diameter is usually different from that of the caudal vena cava. In addition, the common iliac veins can always be followed caudally until the point where they leave the abdomen, unlike a testicular or ovarian vein.

A large amount of free abdominal fluid may hinder ultrasonographic examination of the abdominal vessels, but hyperammonemia in dogs with severe peritoneal effusion cannot possibly be the result of a CPSS or a urea cycle enzyme deficiency. ${ }^{2,19}$ A dog with CPSS would not be expected to have portal hypertension or hypoalbuminemia severe enough to result in formation of a large amount of transudate in the abdominal cavity. ${ }^{5,19} \mathrm{~A}$ small amount of free abdominal fluid is normal in healthy pups and, hence, may also be seen in puppies with CPSSs or urea cycle enzyme deficiency.

Free abdominal fluid was absent in some dogs with sustained portal hypertension in the present study. This is not surprising, in that ascites formation depends not only on the degree of portal pressure but also on the colloid osmotic pressure and albumin concentration of the blood, as well as the effectiveness of the APSSs. ${ }^{2}$ In the dog that had a combination of arterioportal fistulae and an intrahepatic portocaval shunt, ascites and APSSs did not develop because the portocaval shunt did not allow portal hypertension to develop.

No splenomegaly was found in any of the dogs with portal hypertension in the present study. However, it is a common finding in humans and was suggested to be present in dogs as well. ${ }^{48}$ Possibly, splenomegaly does not consistently develop in dogs with APSSs because acquired splenic-renal collateral vessels are the most consistently developed APSSs in dogs, and these collateral veins prevent splenic congestion.

In dogs with a urea cycle enzyme deficiency, a functional disorder is responsible for the hyperammonemia. Thus, the ultrasonographic appearance of the abdominal organs and vessels does not differ from normal.

Scanning time that was necessary to image the anomalous vein or to exclude portosystemic shunting in dogs in the present study was approximately 15 to 30 minutes, which was not substantially different from the time required for routine abdominal ultrasonography. This was possible not only because of the experience and skill of the ultrasonographer and the use of a high-quality ultrasound system, but also because of the use of our systematic scanning protocol ${ }^{23}$ and an extensive knowledge of the anatomy of the various types of possible vascular anomalies. Moreover, an important factor that made the examination times shorter was that the clinician's request was straightforward and based on a high blood ammonia concentration, so that only 3 conditions had to be distinguished ultrasonographically: normal anatomy, CPSS, and portal hypertension.

In sum, results of our study suggest that ultrasonography is a reliable diagnostic method to noninvasively characterize the underlying disease in dogs with hyperammonemia. The cause of APSSs can be established ultrasonographically in dogs with arterioportal fistulae. However, in other instances, histologic examination of a specimen obtained by means of ultrasound-guided liver biopsy is necessary.

${ }^{a}$ Gerritzen-Bruning MJ, Rothuizen J. Sensitivity and specificity of ammonia and bile acids in diagnosing portosystemic shunting (abstr), in Proceedings. "Voorjaarsdagen" Int Vet Congr, Amsterdam, The Netherlands, 2003.

${ }^{b}$ ATL HDI 3000, Advanced Technology Laboratories Ultrasound, Philips Medical Systems, Woerden, The Netherlands.

Cullen JM. Histology of vascular disorders of the canine and feline liver (abstr), in Proceedings. 21st Annu Am Coll Vet Int Med Forum, Charlotte, NC, 2003.

\section{References}

1. Strombeck DR, Meyer DJ, Freedland RA. Hyperammonemia due to a urea cycle enzyme deficiency in two dogs. IAm Vet Med Assoc 1975;166:1109-1111.

2. Johnson SE. Portal hypertension. Part I. Pathophysiology and clinical consequences. Compend Contin Educ Pract Vet 1987;9:741-748.

3. Vitums A. Portosystemic communications in the dog. Acta Anat (Basel) 1959;39:271-299.

4. van den Ingh TSGAM, Rothuizen J, Meyer HP. Portal hypertension associated with primary hypoplasia of the hepatic portal vein in dogs. Vet Rec 1995; 137:424-427.

5. van den Ingh TSGAM, Rothuizen J, Meyer HP. Circulatory disorders of the liver in dogs and cats. Vet Q 1995;17:70-76.

6. Bunch SE, Johnson SE, Cullen JM. Idiopathic noncirrhotic portal hypertension in dogs: 33 cases (1982-1998). IAm Vet Med Assoc 2001;218:392-399.

7. Szatmári V, Németh T, Kótai I, et al. Doppler ultrasonographic diagnosis and anatomy of congenital arterioportal fistula in a puppy. Vet Radiol Ultrasound 2000;41:284-286.

8. Twedt DC. Cirrhosis: a consequence of chronic liver disease. Vet Clin North Am Small Anim Pract 1985;15:151-176.

9. Komtebedde J, Forsyth SF, Breznock EM, et al. Intrahepatic portosystemic venous anomaly in the dog. Perioperative management and complications. Vet Surg 1991;20:37-42.

10. Rothuizen J, van den Ingh TSGAM, Voorhout G, et al. Congenital porto-systemic shunts in sixteen dogs and three cats. J Small Anim Pract 1982;23:67-81.

11. Payne JT, Martin RA, Constantinescu GM. The anatomy and embryology of portosystemic shunts in dogs and cats. Semin Vet Med Surg (Small Anim) 1990;5:76-82.

12. Lamb CR, White RN. Morphology of congenital intrahepatic portocaval shunts in dogs and cats. Vet Rec 1998;142:55-60.

13. Meyer HP, Rothuizen J, Tiemessen I, et al. Transient metabolic hyperammonaemia in young Irish Wolfhounds. Vet Rec 1996; 138:105-107.

14. Hall JA, Allen TA, Fettman MJ. Hyperammonemia associat-

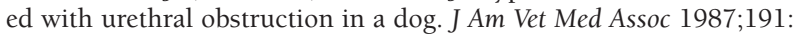
1116-1118.

15. Stone EA, Withrow SJ, Page RL, et al. Ureterocolonic anastomosis in ten dogs with transitional cell carcinoma. Vet Surg 1988; $17: 147-153$

16. Schermerhorn T, Center SA, Dykes NL, et al. Suspected microscopic hepatic arteriovenous fistulae in a young dog. I Am Vet Med Assoc 1997;211:70-74.

17. Wrigley RH, Park RD, Konde LJ, et al. Subtraction portal venography. Vet Radiol 1987;28:208-212.

18. Lamb CR. Ultrasonographic diagnosis of congenital por- 
tosystemic shunts in dogs: results of a prospective study. Vet Radiol Ultrasound 1996;37:281-288.

19. Wrigley RH, Konde LJ, Park RD, et al. Ultrasonographic diagnosis of portacaval shunts in young dogs. $L$ Am Vet Med Assoc 1987;191:421-424.

20. Holt DE, Schelling CG, Saunders HM, et al. Correlation of ultrasonographic findings with surgical, portographic, and necropsy findings in dogs and cats with portosystemic shunts: 63 cases (1987-1993). L Am Vet Med Assoc 1995;207:1190-1193.

21. Tiemessen I, Rothuizen J, Voorhout G. Ultrasonography in the diagnosis of congenital portosystemic shunts in dogs. Vet $Q$ 1995; 17:50-53.

22. Nyland TG, Mattoon JS, Herrgesell EJ, et al. Liver. In: Nyland TG, Mattoon JS, eds. Small animal diagnostic ultrasound. 2nd ed. Philadelphia: WB Saunders Co, 2002;93-127.

23. Szatmári V, Rothuizen J, Voorhout G. Standard planes for ultrasonographic examination of the portal system in dogs. IAm Vet Med Assoc 2004;224:713-716.

24. Wolschrijn CF, Mahapokai W, Rothuizen J, et al. Gauged attenuation of congenital portosystemic shunts: results in 160 dogs and 15 cats. Vet $Q$ 2000;22:94-98.

25. Rothuizen J, van den Ingh TGSAM. Rectal ammonia tolerance test in the evaluation of portal circulation in dogs with liver disease. Res Vet Sci 1982;33:22-25.

26. Meyer HP, Rothuizen J, van den Brom WE, et al Quantitation of portosystemic shunting in dogs by ultrasound-guided injection of 99MTc-macroaggregates into a splenic vein. Res Vet Sci 1994;57:58-62.

27. Vitums A. Portal vein in the dog. Zbl Vet Med 1959;7:723-741.

28. Szatmári V, van Sluijs FJ, Rothuizen J, et al. Ultrasonographic assessment of hemodynamic changes in the portal vein during surgical attenuation of congenital extrahepatic portosystemic shunts in dogs. I Am Vet Med Assoc 2004;224:395-402.

29. Foster DN, Herlinger H, Miloszewski KJ, et al. Hepatofugal portal blood flow in hepatic cirrhosis. Ann Surg 1978;187:179-182.

30. Wachsberg RH, Bahramipour P, Sofocleous CT, et al. Hepatofugal flow in the portal venous system: pathophysiology, imaging findings, and diagnostic pitfalls. Radiographics 2002;22:123-140.

31. Suter PF. Portal vein anomalies in the dog: their angiographic diagnosis. LAm Vet Radiol Soc 1975;16:84-97.

32. Szatmári V, van Sluijs FJ, Rothuizen J, et al. Intraoperative ultrasonography of the portal vein during attenuation of intrahepatic portocaval shunts in dogs. LAm Vet Med Assoc 2003;222:1086-1092.

33. DeMarco JA, Center SA, Dykes N, et al. A syndrome resem- bling idiopathic noncirrhotic portal hypertension in 4 young Doberman Pinschers. IVet Intern Med 1998;12:147-156.

34. McEntee MF, Wright KN, Wanless I, et al. Noncirrhotic portal hypertension and nodular regenerative hyperplasia of the liver in dogs with mucopolysaccharidosis type I. Hepatology 1998; 28:385-390.

35. Rothuizen J. Liver research group considers vascular hepatic diseases. ISmall Anim Pract 2002;43:100-101.

36. Schermerhorn T, Center SA, Dykes NL, et al. Characterization of hepatoportal microvascular dysplasia in a kindred of Cairn Terriers. I Vet Intern Med 1996;10:219-230.

37. Phillips L, Tappe J, Lyman R, et al. Hepatic microvascular dysplasia in dogs. Prog Vet Neurol 1996;7:88-96.

38. Allen L, Stobie D, Mauldin GN, et al. Clinicopathologic features of dogs with hepatic microvascular dysplasia with and without portosystemic shunts: 42 cases (1991-1996). L Am Vet Med Assoc 1999;214:218-220.

39. Christiansen JS, Hottinger HA, Allen L, et al. Hepatic microvascular dysplasia in dogs: a retrospective study of 24 cases (1987-1995). L Am Anim Hosp Assoc 2000;36:385-389.

40. Schaeffer IG, Kirpensteijn J, Wolvekamp WT, et al. Hepatic arteriovenous fistulae and portal vein hypoplasia in a Labrador Retriever. L Small Anim Pract 2001;42:146-150

41. Sterczer A, Gaál T, Perge E, et al. Chronic hepatitis in the dog-a review. Vet $Q$ 2001;23:148-152.

42. Lamb CR. Ultrasonography of portosystemic shunts in dogs and cats. Vet Clin North Am Small Anim Pract 1998;28: 725-753.

43. Thurmon JC, Tranquilli WJ, Benson GJ. Considerations for general anesthesia. In: Thurmon JC, Tranquilli WJ, Benson GJ, eds. Lumb \& Jones' veterinary anaesthesia. Baltimore: Lea \& Febiger, 1996; $5-34$.

44. Szatmári V, van den Ingh TSGAM, Fenyves B, et al. Portal hypertension in a dog due to circumscribed fibrosis of the wall of the extrahepatic portal vein. Vet Rec 2002;150:602-605.

45. Boothe HW, Howe LM, Edwards JF, et al. Multiple extrahepatic portosystemic shunts in dogs: 30 cases (1981-1993). LAm Vet Med Assoc 1996;208:1849-1854.

46. Valentine RW, Carpenter JL. Spleno-mesenteric-renal venous shunt in two dogs. Vet Pathol 1990;27:58-60.

47. Laborda J, Gimeno M, Dominguez L, et al. Anomalous caudal vena cava in the dog. Vet Rec 1996;138:20-21.

48. Finn-Bodner ST, Hudson JA. Abdominal vascular sonography. Vet Clin North Am Small Anim Pract 1998;28:887-942. 